\title{
Spindle Epithelial Tumor with Thymus-Like Differentiation (SETTLE): A Distinctive Malignant Thyroid Neoplasm with Significant Metastatic Potential
}

\author{
Wah Cheuk, M.D., Avram A. Jacobson, M.D., John K. C. Chan, M.D. \\ Department of Pathology, Queen Elizabeth Hospital, Hong Kong (WC, JKCC) and Kaiser Permanente \\ Foundation Hospitals, Los Angeles, California (AAJ)
}

\begin{abstract}
Spindle epithelial tumor with thymus-like differentiation (SETTLE) is a very rare tumor of the thyroid believed to be derived from branchial pouch or thymic remnants and showing primitive thymic differentiation. Although this tumor is prone to develop delayed blood-borne metastases, the metastatic risk is unclear because the case reports in the recent literature had very short follow-up periods. We report one case, the oldest patient reported so far, who had been followed up until death. The 59-year-old man had an enlarged thyroid for all of his adult life, and presented with recent rapid enlargement of the thyroid. Neck exploration revealed a hard tumor in the right lobe, with adhesion to sternothyroid muscle. Histologic examination showed an invasive biphasic neoplasm traversed by sclerotic septa. Tight to loose fascicles of blandlooking spindly cells were intimately intermingled with tubulopapillary structures, diagnostic of the SETTLE. This patient developed left pulmonary metastases at $\mathbf{2}$ years and subsequently developed bilateral pulmonary and widespread metastases. He died 8 years after initial presentation. This case illustrates the protracted clinical course of the tumor, and survival for many years despite the occurrence of metastases. Review of the literature shows that SETTLE occurs predominantly in young patients with a median age of 15 years and male predominance. There is a significant metastatic rate of $71 \%$ for patients with more than 5 years of follow-up in spite of the otherwise indolent nature of the tumor.
\end{abstract}

\footnotetext{
Copyright (C) 2000 by The United States and Canadian Academy of Pathology, Inc.

VOL. 13, NO. 10, P. 1150, 2000 Printed in the U.S.A.

Date of acceptance: May 11, 2000.

Address reprint requests to: Wah Cheuk, M.D., Department of Pathology, Queen Elizabeth Hospital, Wylie Road, Kowloon, Hong Kong. Fax 85223852455 .
}

KEY WORDS: Immunohistochemistry, Metastasis, SETTLE, Spindle cell tumor, Thyroid tumor.

Mod Pathol 2000;13(10):1150-1155

Spindle epithelial tumor with thymus-like differentiation (SETTLE) is a very rare thyroid tumor formally characterized by Chan and Rosai (1) in 1991 as a tumor of the neck showing thymic or related branchial pouch differentiation. Isolated reports of this tumor entity had previously been reported under a variety of designations, such as thyroid spindle cell tumor with mucous cyst, malignant teratoma, thyroid thymoma in childhood, and "unusual thyroid tumor in a child" (2-6). SETTLE occurs in young patients, with indolent growth and a tendency to develop delayed blood-borne metastases. Since the report of Chan and Rosai (1), a number of case reports have appeared in the literature, but all of them are flawed by short follow-up periods and incomplete compilation of the previously reported cases (7-12). Consequently, the malignant potential of this tumor may not be fully evident from these data. We herein report one case, the oldest patient reported so far, who had been followed up until death. The clinicopathologic features of all reported cases in the English-language literature are also reviewed.

\section{CASE REPORT}

A 59-year-old man had an enlarged thyroid for all of his adult life. Over a period of 6 weeks before hospitalization, the thyroid was noted to enlarge rapidly. Eighteen years ago, the patient had bilateral seminoma of the testes treated by bilateral orchidectomy and irradiation to the groin area, and had remained tumor free. One year ago, bilateral breast removal was performed because of gynecomastia.

Physical examination revealed an enlarged and hard thyroid gland, most notably in the right lobe. There were no palpable lymph nodes. A thyroid 


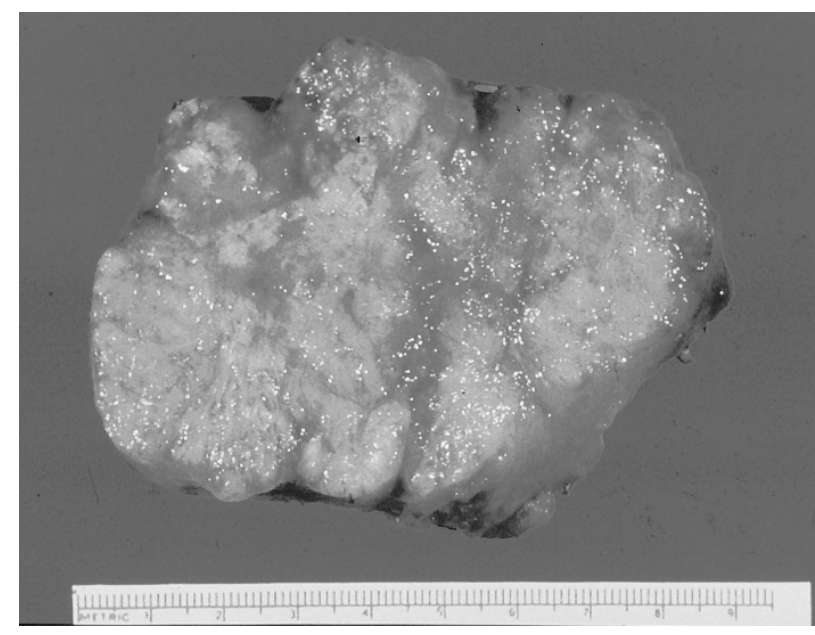

FIGURE 1. Thyroid resection specimen. The thyroid parenchyma was entirely replaced by grayish-tan tumor. A vague lobular pattern was discernible.

scan showed a large cold nodule in the right lobe. Systemic investigations did not yield significant findings.

Neck exploration was performed, revealing that the right lobe of the thyroid was hard and adherent to the right sternothyroid muscle. There was no evidence of nodal metastasis on either side of the neck. Right hemithyroidectomy was carried out. The histologic diagnosis rendered at that time (1970) was medullary thyroid carcinoma, and had recently been revised to SETTLE. Two years after surgical excision, the patient developed metastasis to the left lung, requiring left upper lobectomy. Subsequently, bilateral pulmonary metastases were found, and the patient was given chemotherapy.

The patient died of disseminated tumor 8 years after initial diagnosis. At autopsy, metastatic deposits were found in the left pleural surfaces, mediastinal lymph nodes, left main bronchus, both lungs, peri-esophageal areas, pancreas, and kidney.

\section{Pathologic Examination}

\section{Gross and histologic appearances}

The thyroid tumor was partially circumscribed, measuring $9 \times 6 \times 3.5 \mathrm{~cm}$. The external surface was bosselated, and the cut surface showed grayish-tan tumor with vague lobulation (Fig. 1).

Histologic examination showed an invasive, highly cellular neoplasm traversed by irregular thin and thick sclerotic bands giving rise to multiple
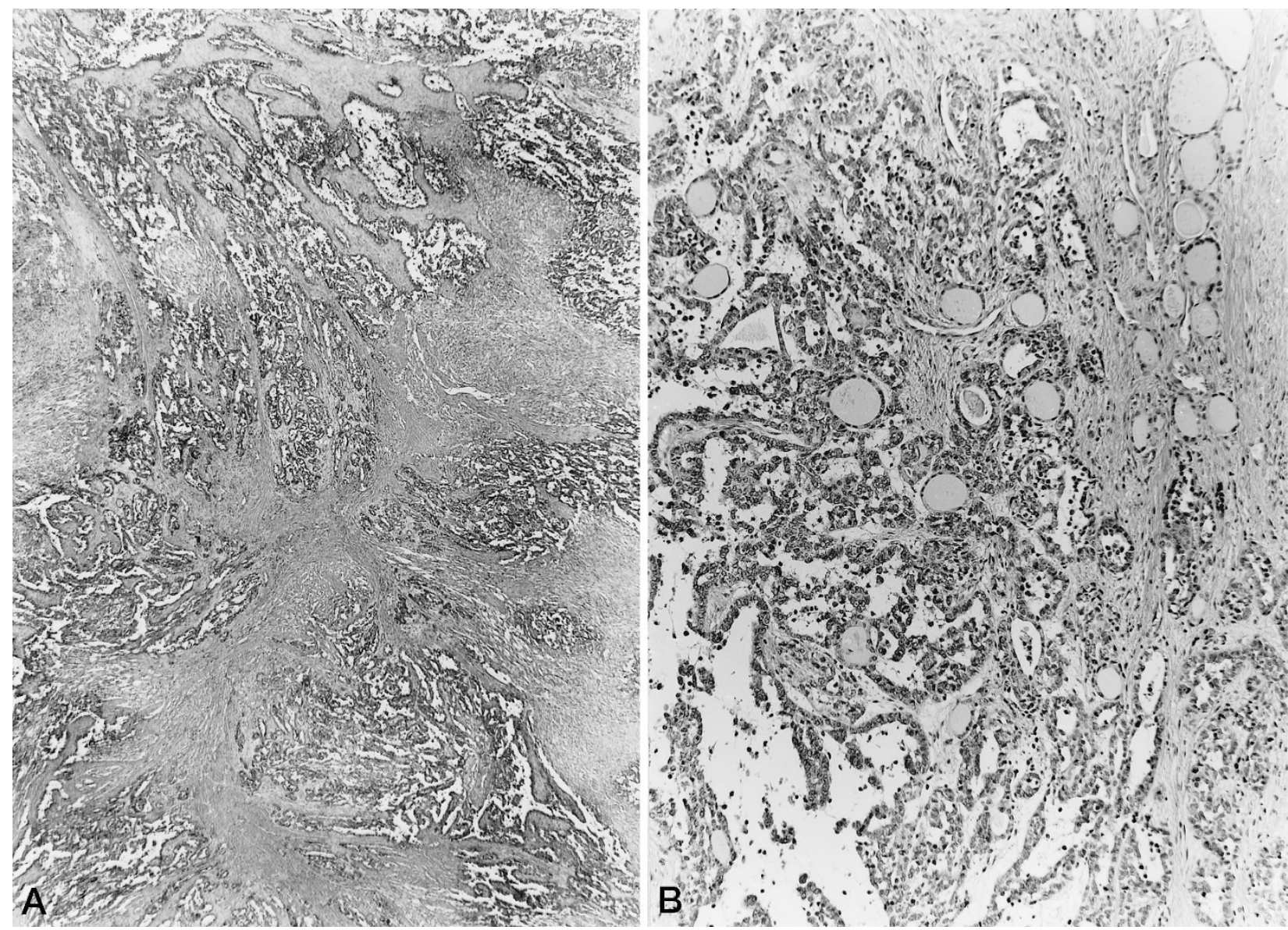

FIGURE 2. Low magnification view of tumor. A, the tumor was traversed by irregular fibrous bands giving rise to a lobulated pattern. Note the complex tubulopapillary pattern of the glandular component (hematoxylin and eosin stain; original magnification, $6 \times$ ). B, thyroid follicles were entrapped in the invasive fronts of the tumor (hematoxylin and eosin stain; original magnification, $30 \times$ ). 


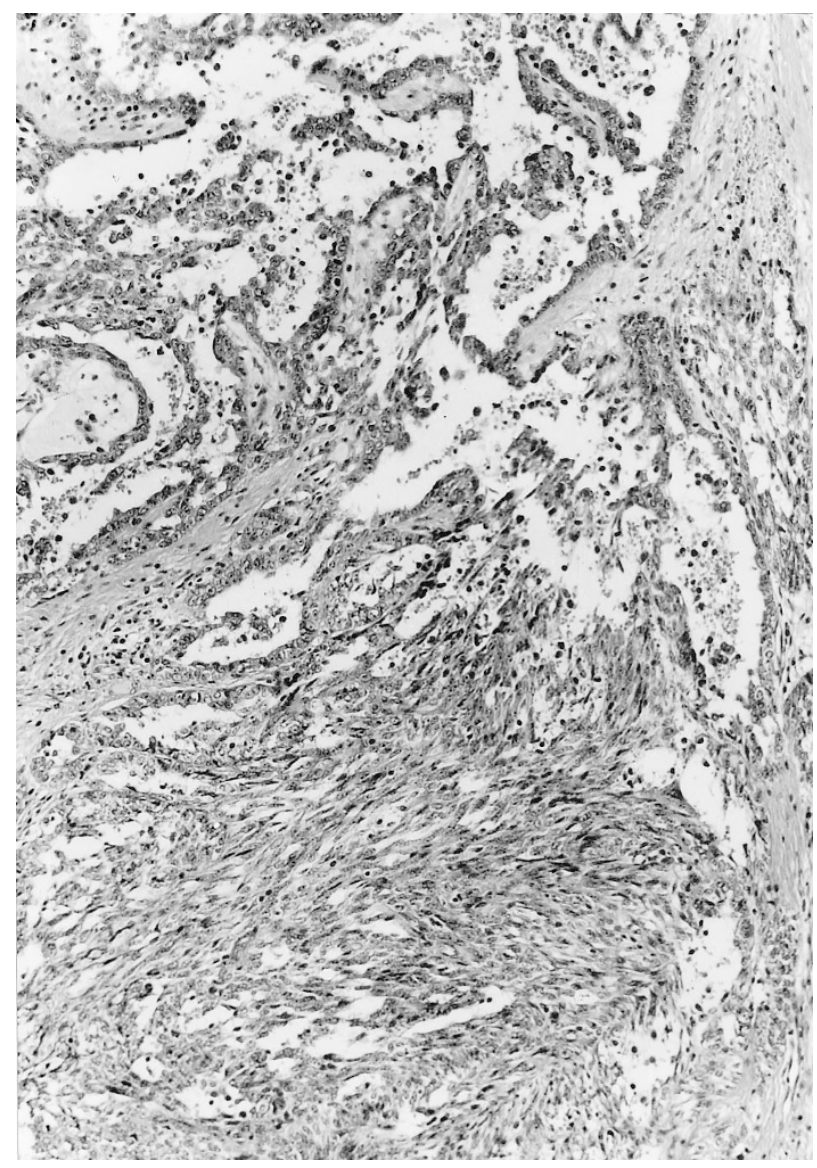

FIGURE 3. Biphasic pattern of tumor. Streaming fascicles of spindly cells merged imperceptibly with tubulopapillary structures (hematoxylin and eosin stain; original magnification, $60 \times$ ).

vague lobules (Fig. 2). The tumor had a biphasic pattern characterized by merging of cytologically bland and mitotically inactive spindle cells with tubulopapillary epithelial structures (Fig. 3). The former component accounted for approximately $40 \%$ of the entire tumor.

The spindle cells possessed elongated or oval nuclei with delicate chromatin and inconspicuous nucleoli (Fig. 4). Nuclear pleomorphism was minimal and mitotic figures were rare. The scanty to moderate amount of faintly eosinophilic cytoplasm was ill defined. The spindle cells formed tight to loose streaming fascicles, with focal reticulated pattern produced by intercellular edema. They blended imperceptibly with glands and narrow tubulopapillary structures that were lined by bland-looking cuboidal to columnar cells. There were some areas where one of the two components dominated. Lymphocytes were sparse. Focal lymphovascular permeation by tumor cells was present.

The metastatic foci in various organs generally retained the morphology of the original tumor (Fig. 5). However, foci of coagulative necrosis were noted in the metastatic tumor in the kidney.

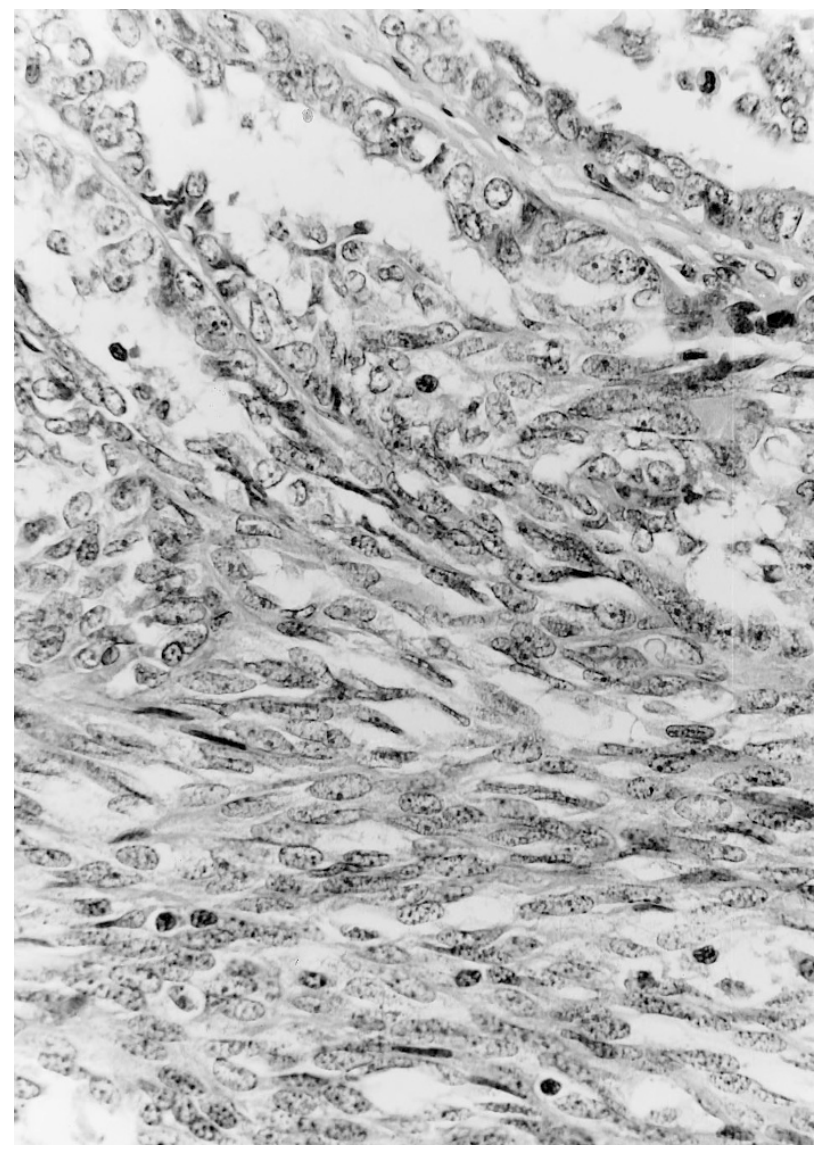

FIGURE 4. High magnification view of the tumor. The spindle cells showed a streaming pattern and possessed oval vesicular nuclei with minimal nuclear atypia. The glandular structures were lined by blandlooking cuboidal to columnar cells (hematoxylin and eosin stain; original magnification, $120 \times$ ).

\section{Immunohistochemistry}

Immunohistochemical staining was performed using the VENTANA automated immunostainer with a labeled streptavidin-biotin peroxidase detection system. The spindle cells as well as the glandular cells showed positive staining for epithelial markers (Fig. 6), but negative staining for thyroglobulin, calcitonin, S-100 protein, and actin (Table $1)$. The staining for $34 \beta \mathrm{E} 12$ was the strongest, whereas staining with MNF-116, CAM5.2, and EMA was more patchy. CD5 was negative. There were no TdT-positive lymphocytes.

\section{DISCUSSION}

SETTLE, a rare primary tumor of the thyroid, is composed of spindle cells of epithelial nature forming fascicles, merging into glandular structures taking the form of tubules, papillae, and cystic spaces. In some cases, cysts or glands lined by mucinous or respiratory epithelium may be present. Rare cases may be predominantly monophasic, with spindle cell predominance (10). It has been speculated that SETTLE represents a neoplasm arising from bran- 


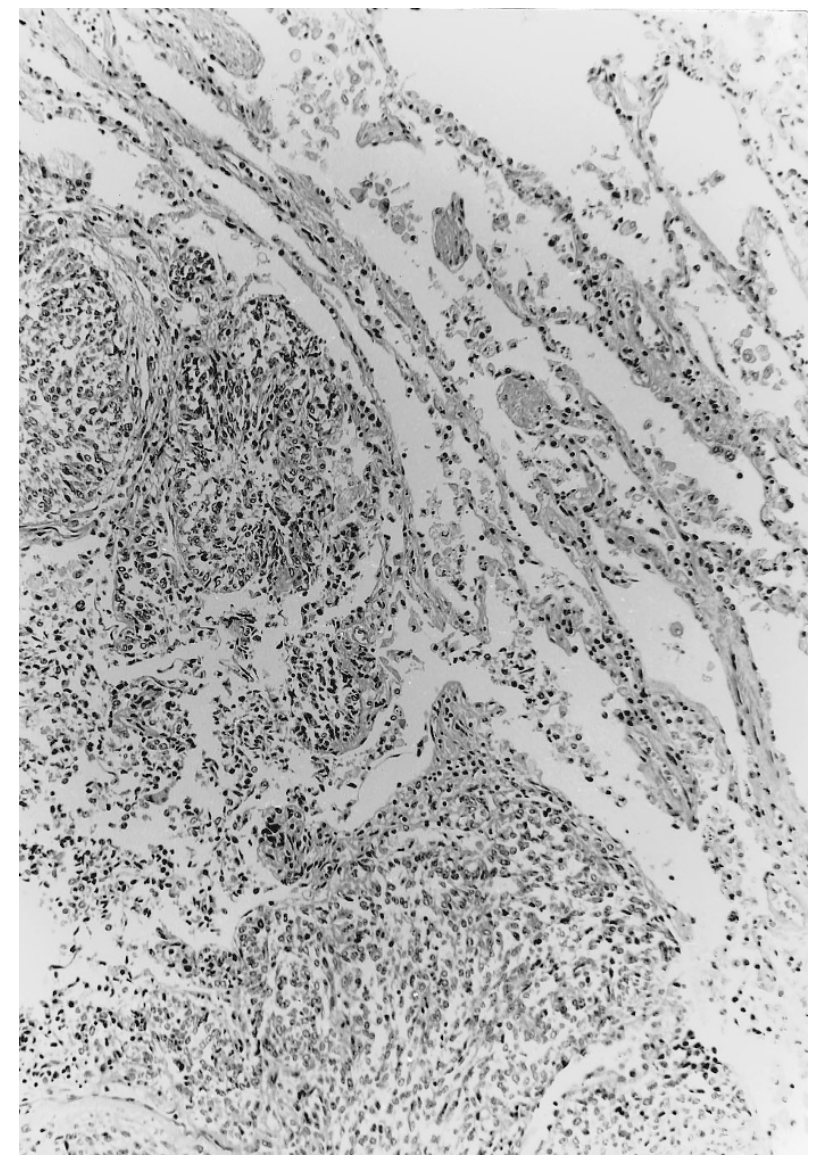

FIGURE 5. Metastatic tumor in the lung (hematoxylin and eosin stain; original magnification, $30 \times$ ).

chial pouch remnants or ectopic thymus, showing possible differentiation towards embryonic thymus (thymoblastoma) (1). In one case, the SETTLE arose in association with epithelium-lined cysts of possible branchial pouch derivation (8). Nonetheless, so far convincing proof of thymic differentiation is lacking: (1) CD20, a B-cell-associated marker expressed in the epithelial cells in a proportion of spindle cell (type A) thymomas (13), is consistently negative; (2) CD5, a leukocyte marker expressed in a high proportion of thymic carcinomas, is negative in the two cases on which this marker has been studied (one previously reported and the current case) (14); and (3) TdT+ immature T lymphocytes

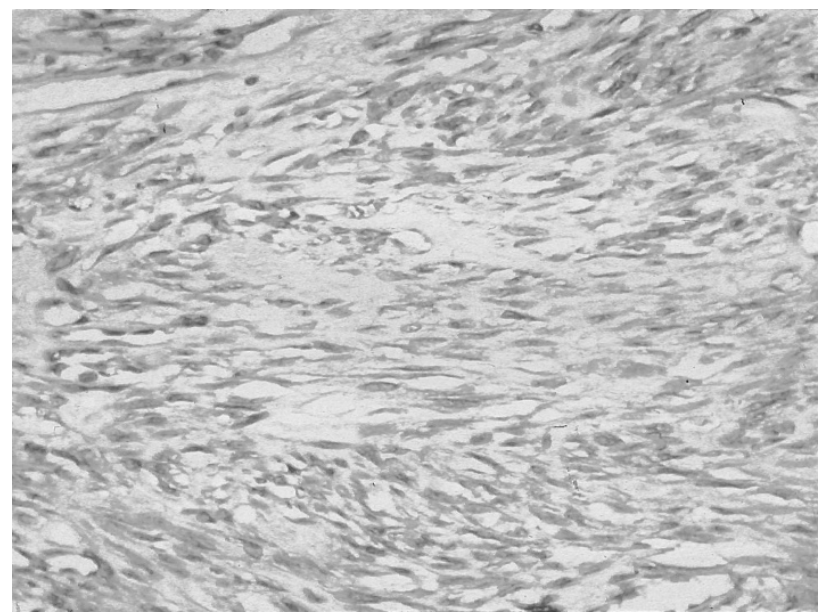

FIGURE 6. Immunostaining for pan-cytokeratin in the primary tumor. Diffuse staining was evident in the neoplastic spindly cells (pancytokeratin stain; original magnification, $60 \times$ ).

that typically accompany thymomas have not been found in this neoplasm. Thus, the histogenesis of SETTLE remains elusive, and it is even possible that it represents a primitive thyroid neoplasm ("thyroblastoma") instead. Notwithstanding the uncertainties on the nature of the tumor, SETTLE is undoubtedly a distinctive tumor type of the thyroid different from other known thyroid neoplasms. When more is known about the patterns of gene expression in the thyroid and thymus, analysis of the gene expression patterns in SETTLE using microarray chips can potentially shed light on the histogenesis of the tumor.

In SETTLE, immunohistochemical staining reveals positivity of the spindly tumor cells for epithelial markers, consistent with the ultrastructural observation of tonofilaments and desmosomes $(7-9,12)$. Interestingly, the spindle cells show strong staining with $34 \beta E 12$, indicating expression of high molecular weight cytokeratin as commonly seen in squamous cells; this feature is also observed in two additional examples of SETTLE that we have studied (results not shown). Su et al. (9) reported one case of SETTLE that demonstrated myoepithelial phenotype including immunoreactivity for smooth muscle actin and ultrastructural evidence

TABLE 1. Immunohistochemical Findings

\begin{tabular}{|c|c|c|c|}
\hline Antibody & Source & Dilution & Result \\
\hline Pan-cytokeratin (MNF 116) & Dakopatts & $1: 50$ & + (patchy) \\
\hline Low molecular weight cytokeratin (CAM5.2) & Becton-Dickinson & $1: 8$ & + (patchy) \\
\hline High molecular weight cytokeratin (34ßE12) & Dakopatts & 1:100 & + (diffuse) \\
\hline Epithelial membrane antigen (E29) & Dakopatts & $1: 1000$ & + (patchy) \\
\hline CD5 (NCL-CD5-4C7) & Novacastra & $1: 10$ & - \\
\hline Thyroglobulin (Dako-Tg6) & Dakopatts & $1: 100$ & - \\
\hline Calcitonin (polyclonal) & Dakopatts & $1: 800$ & - \\
\hline S-100 protein (polyclonal) & Dakopatts & $1: 1000$ & - \\
\hline Muscle-specific actin (HHF35) & Enzo Biochem & $1: 3$ & - \\
\hline Terminal deoxynucleotide transferase (TdT) (polyclonal) & Supertechs & 1:10 & - \\
\hline
\end{tabular}




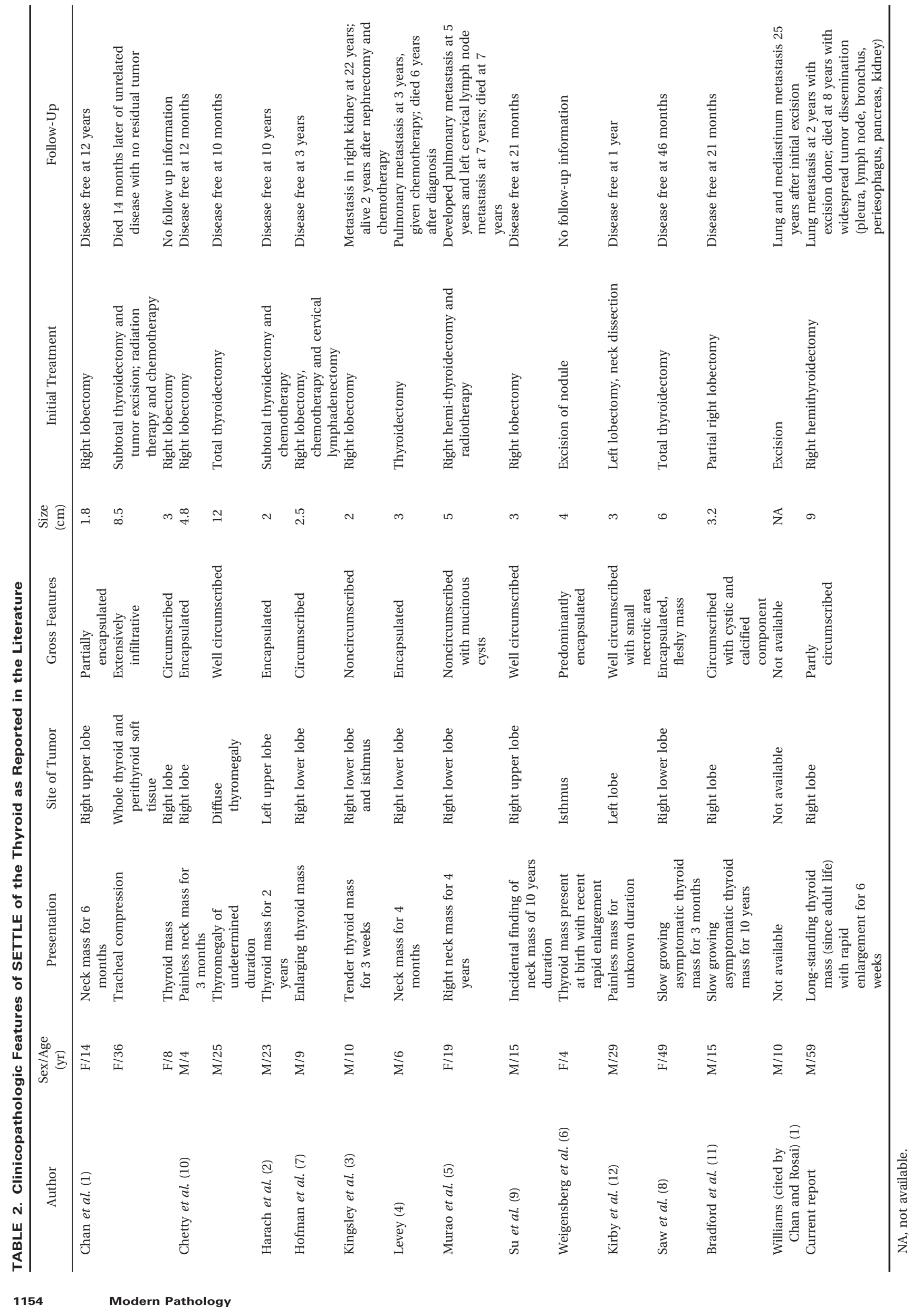


of cytoplasmic filaments with dense bodies. However, this feature is not seen in other cases of SETTLE.

From the available data in the literature (17 cases), SETTLE occurs predominantly in children, adolescents, and young adults, with a median age of 15 years (range, 4 to 59 years) at presentation (Table 2 ). The male to female ratio is $1.8: 1$. The patient included in this report is the oldest reported so far, but the tumor might have originated in early adulthood because the thyroid mass was present for all of his adult life. In the literature, there are at least four other patients who had the thyroid mass noticed for four or more years, attesting to the generally slow growth of the neoplasm. Sometimes rapid enlargement of the lesion is noted in the longstanding thyroid mass.

SETTLE pursues an indolent course, but metastasis may not occur until many years after diagnosis (mean, 11.4 years; median, 5 years). The overall rate of metastasis is $33 \%(5 / 15)$ among the reported cases with follow-up information, but increases to a significant $71 \%(5 / 7)$ if analysis is made only on patients who had more than 5 years of follow-up. Among them, two patients developed metastasis more than 20 years after the initial diagnosis. The preferential site of metastasis is the lung. Thus, long-term follow-up with particular attention to monitoring the lungs with imaging studies is advisable. The previous reports with short follow-up periods impart a false picture of "benignity" to the tumor. Another characteristic feature of SETTLE is that despite development of metastases, long survival for many years is still possible, attesting to the indolence of the tumor, as exemplified by the current case and the case reported by Levey (4).

SETTLE must be distinguished from sarcomatoid anaplastic carcinoma because of the very different prognosis. The latter is characterized clinically by rapid tumor growth and early tumor mortality within 1 year, and histologically by overt nuclear atypia, frequent mitoses, and necrosis. Medullary carcinoma can have spindle cells and gland-like structures, and thus may mimic SETTLE, but the fibrovascular septa are much more prominent and the tumor cells have stippled chromatin and granular cytoplasm. Immunostaining for calcitonin and chromogranin is confirmatory of the diagnosis. SETTLE differs from thymoma in lacking the characteristic jigsaw puzzle-like lobulation, TdTpositive thymocytes, and ultrastructurally interdigitating cell processes. Furthermore, the extensive glandular pattern of SETTLE is not found in thymoma. Distinction of SETTLE from synovial sarcoma is most difficult, but the spindle cells in synovial sarcoma generally show only patchy cytokeratin immunoreactivity and tonofilaments are not found ultrastructurally.

In conclusion, this report documents the complete follow-up of an example of SETTLE, and provides the most comprehensive review on the behavioral features of this tumor, putting its metastatic potential into proper perspective.

\section{REFERENCES}

1. Chan JK, Rosai J. Tumors of the neck showing thymic or related branchial pouch differentiation: a unifying concept. Hum Pathol 1991;22:349-67.

2. Harach HR, Saravia Day E, Franssila KO. Thyroid spindle-cell tumor with mucous cysts. An intrathyroid thymoma? Am J Surg Pathol 1985;9:525-30.

3. Kingsley DPE, Elton A, Bennett MH. Malignant teratoma of the thyroid: case report and a review of the literature. Br J Cancer 1968;22:7-11.

4. Levey M. An unusual thyroid tumor in a child. Laryngoscope 1976;86:1864-8.

5. Murao T, Nakanishi M, Toda K, Honishi H. Malignant teratoma of the thyroid gland in an adolescent female. Acta Pathol Jpn 1979;29:109-17.

6. Weigensberg C, Dalsley H, Asa SL, Bedard YC, Mullen JBM. Thyroid thymoma in childhood. Endocr Pathol 1990;1:123-7.

7. Hofman P, Mainguene C, Michiels JF, Pages A, Thyss A. Thyroid spindle epithelial tumor with thymus-like differentiation (the "SETTLE" tumor). An immunohistochemical and electron microscopic study. Eur Arch Otorhinolaryngol 1995; 252:316-20.

8. Saw D, Wu D, Chess Q, Shemen L. Spindle epithelial tumor with thymus-like element (SETTLE), a primary thyroid tumor. Int J Surg Pathol 1997;4:169-74.

9. Su L, Beals T, Bernacki EG, Giordano TJ. Spindle epithelial tumor with thymus-like differentiation: a case report with cytologic, histologic, immunohistologic, and ultrastructural findings. Mod Pathol 1997;10:510-4.

10. Chetty R, Goetsch S, Nayler S, Cooper K. Spindle epithelial tumour with thymus-like element (SETTLE): the predominantly monophasic variant. Histopathology 1998;33:71-4.

11. Bradford CR, Devaney KO, Lee JI. Spindle epithelial tumor with thymus-like differentiation: a case report and review of the literature. Otolaryngol Head Neck Surg 1999;120:603-6.

12. Kirby PA, Ellison WA, Thomas PA. Spindle epithelial tumor with thymus-like differentiation (SETTLE) of the thyroid with prominent mitotic activity and focal necrosis. Am J Surg Pathol 1999;23:712-6.

13. Chilosi M, Castelli P, Martignoni G, Pizzolo G, Montresor E, Facchetti F, et al. Neoplastic epithelial cells in a subset of human thymomas express the B cell-associated CD20 antigen. Am J Surg Pathol 1992;16:988-97.

14. Dorfman DM, Shahsafaei A, Chan JK. Thymic carcinomas, but not thymomas and carcinomas of other sites, show CD5 immunoreactivity. Am J Surg Pathol 1997;21:936-40. 